\title{
Editorial
}

\section{Advances in Mobile Video Networking}

\author{
Qi Wang, ${ }^{1}$ Guojun Wang, ${ }^{2}$ Christos Grecos, ${ }^{3}$ and Ansgar Gerlicher ${ }^{4}$ \\ ${ }^{1}$ University of the West of Scotland, Paisley PA1 2BE, UK \\ ${ }^{2}$ Guangzhou University, Guangzhou 510006, China \\ ${ }^{3}$ Central Washington University, 400 E. University Way, Ellensburg, WA 98926, USA \\ ${ }^{4}$ Stuttgart Media University, Hochschule der Medien Nobelstraße 10, 70569 Stuttgart, Germany
}

Correspondence should be addressed to Qi Wang; qi.wang@uws.ac.uk

Received 21 July 2016; Accepted 21 July 2016

Copyright (C) 2016 Qi Wang et al. This is an open access article distributed under the Creative Commons Attribution License, which permits unrestricted use, distribution, and reproduction in any medium, provided the original work is properly cited.

Video applications have been increasingly dominating worldwide mobile network traffic in the last years. The growing popularity of smart phones and other mobile devices, ondemand and surveillance video services, multimedia social networking, the latest advances in video coding and transmission, and the significant increase in mobile network capacity have all contributed to this global phenomenon. Both challenges and opportunities have emerged in multiple research disciplines involving video signal processing, 4G such as LTE (Long Term Evolution) and even 5G mobile networks, mobile cloud computing, Big Visual Data, mobile user experience, and so on. Research in these leading-edge areas has been gaining accelerated global momentum in both developing and developed economies.

Therefore, it is high time to call for contributions to further stimulate the continuing efforts to resolve the intrinsic conflicts between resource-demanding video applications and resource-constrained mobile networks, optimise video networking performance in mobile networks and achieve improved Quality of Experience (QoE) for end users, and leverage cloud computing and other cutting-edge technologies and standards in supporting adaptive, secure, energyefficient, and reliable video delivery in various mobile systems. This special issue attracted numerous submissions, out of which five papers have been accepted to be published. These accepted papers are expected to highlight some of the important aspects outlined above. It is the pleasure for the guest editorial team to introduce these papers as follows.

The first paper entitled "Energy-Efficient Transmission Strategy by Using Optimal Stopping Approach for Mobile Networks" by Y. Peng et al. contributes to greener video delivery in mobile networks. An optimised distributed opportunistic scheduling algorithm is proposed to maximise the usage of good quality slots of the time-varying wireless transmission channels to improve video data delivery ratio and thus energy efficiency, whilst taking into account the delay constraint for video applications.

The second paper "Network-Aware Reference Frame Control for Error-Resilient H.264/AVC Video Streaming Service" by H.-S. Gang et al. focuses on enhancing the reliability of video streaming in error-prone networks such as mobile/wireless networks whilst making a trade-off between error resilience and coding efficiency. In particular, error propagation across video frames caused by video packet loss or corruption is effectively mitigated, through controlling the number of reference video frames based on the awareness of network channel status.

In the third paper, "A Framework for QoE-Aware 3D Video Streaming Optimisation over Wireless Networks" by I. Politis et al., the challenging topic of $3 \mathrm{D}$ video streaming in LTE networks with good perceived quality is addressed. To model the QoE of end users, machine learning techniques are utilised and parameters from multiple layers are monitored and collected. In addition, a media-aware proxy is introduced to the proposed system, integrated with the QoE controller, to achieve QoE-aware, optimised 3D video transmission.

The fourth paper, "A Low Energy Consumption Storage Method for Cloud Video Surveillance Data Based on SLA Classification" by Y. Xiong et al., also deals with the energy efficiency in a video system although the main concern is on the storage side other than the transmission side as in the first paper. The targeted application is Cloud Video Surveillance, 
and the design objective is to reduce the power consumption without compromising the Service Level Agreement (SLA) for users to access the stored surveillance video data in the cloud on demand in a timely fashion. Based on classifying the access time periods and thus virtual machines and storage nodes, the proposed system reduces overall power consumption in the data centre by placing selected nodes into an energy-saving mode.

In the final paper "A Novel Adaptation Method for HTTP Streaming of VBR Videos over Mobile Networks," $\mathrm{H}$. T. Le et al. investigate adaptive on-demand transmission of variable bitrate (VBR) videos through HTTP streaming. Their adaptation scheme is designed to be tolerant to large variations of video bitrate, featured with a buffer-based algorithm that considers smoothed throughput estimate, representative bitrate, and instant bitrate.

\section{Acknowledgments}

The guest editorial team would like to thank all the authors for submitting their manuscripts to this special issue and all the invited reviewers for their time and constructive feedback. Moreover, the team appreciate the staff of this journal for their continuous support and patience in creating this special issue.

Qi Wang

Guojun Wang

Christos Grecos

Ansgar Gerlicher 

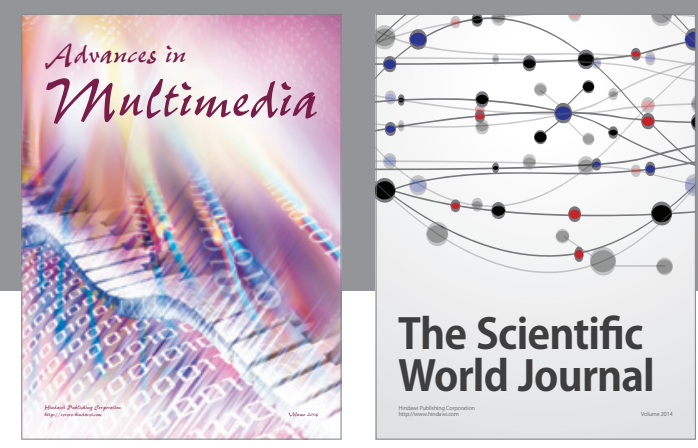

The Scientific World Journal
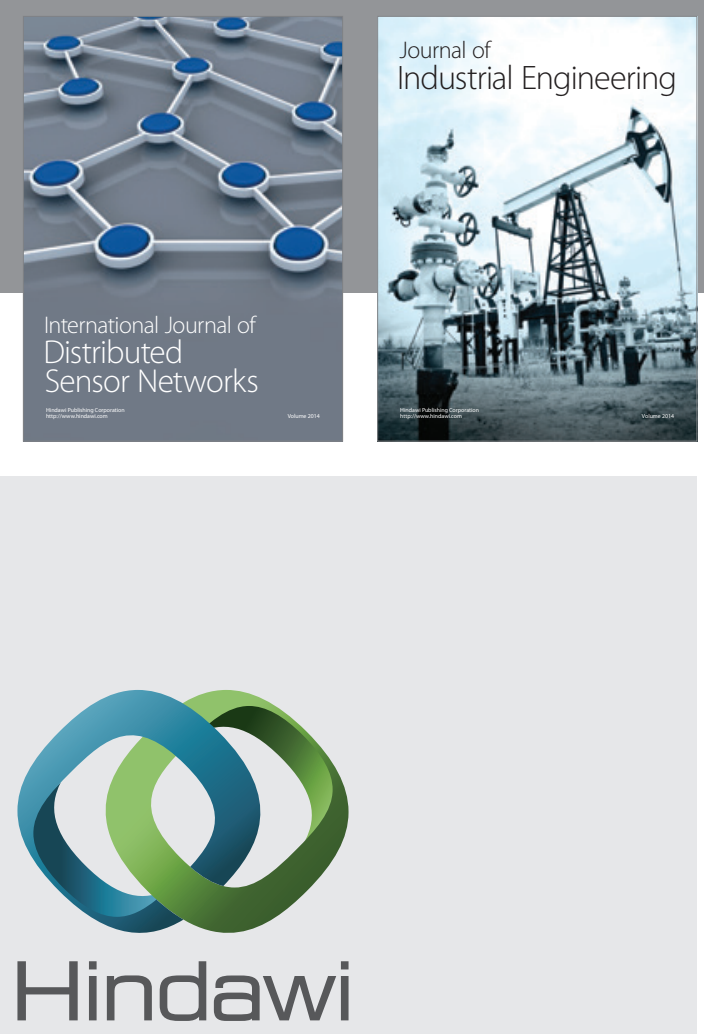

Submit your manuscripts at

http://www.hindawi.com

\section{Computer Networks} and Communications
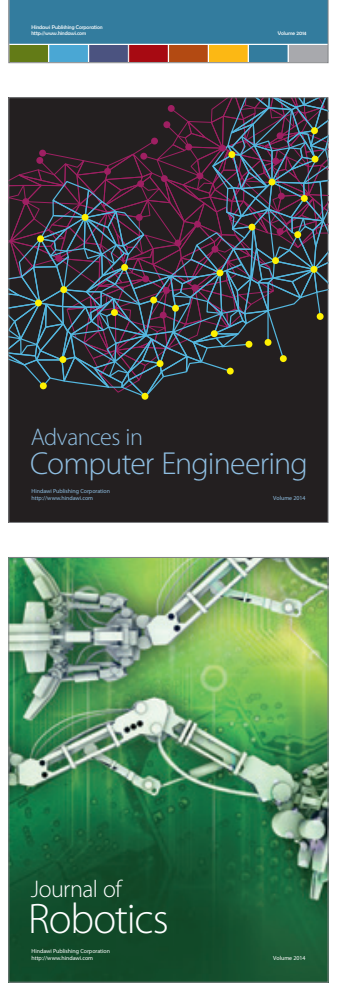
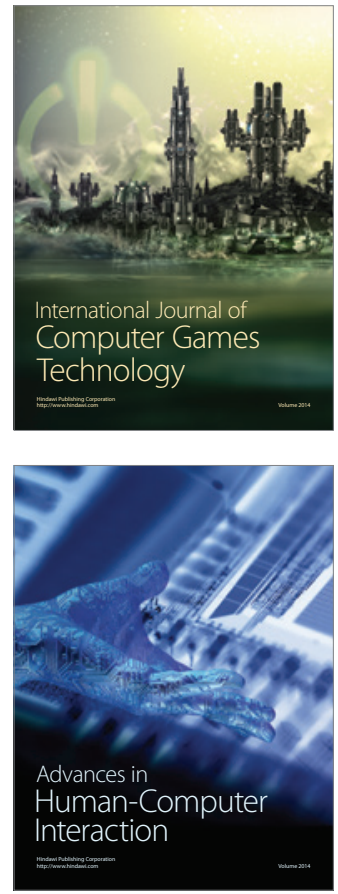
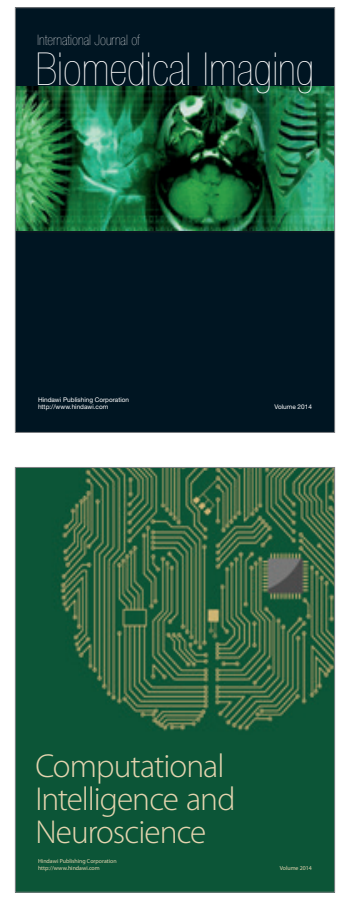
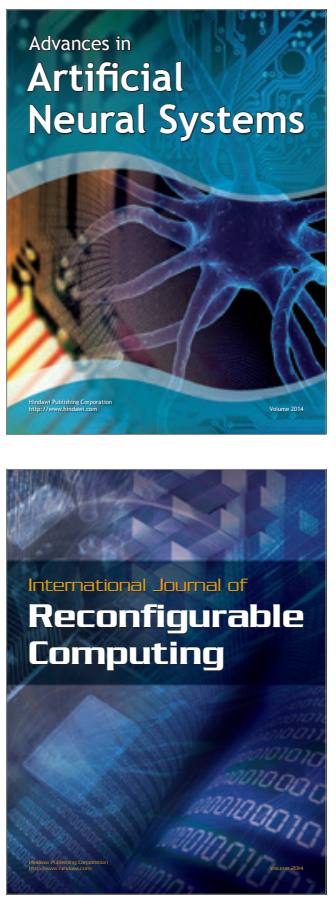
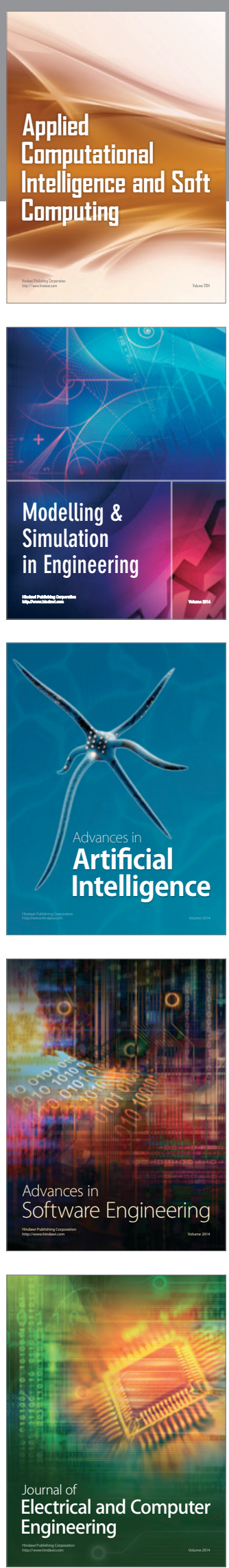